American Journal of Agricultural and Biological Sciences 3 (2): 502-510, 2008

ISSN 1557-4989

(C) 2008 Science Publications

\title{
Processing of Aloe Vera Leaf Gel: A Review
}

\author{
C.T. Ramachandra and P. Srinivasa Rao \\ Department of Agricultural and Food Engineering, \\ Indian Institute of Technology, Kharagpur-721302, India
}

\begin{abstract}
Proper scientific investigations on Aloe vera have gained more attention over the last decade due to its reputable, medicinal, pharmaceutical and food properties. Some publications have appeared in reputable scientific journals that have made appreciable contributions to the discovery of the functions and utilizations of Aloe vera lacking processing of leaf gel. Present processing techniques aims at producing best quality aloe products but end aloe products contain very little or virtually no active ingredients. Hence, appropriate processing techniques should be employed during processing in order to extend the use of aloe vera gel. Further research needs to be done to unravel the myth surrounding the biological activity and the exploitation of aloe constituents.
\end{abstract}

Key words: Cold process, Qmatrix process, whole leaf process, desiccant air dehydration, time temperature, sanitation process

\section{INTRODUCTION}

Aloe vera (Aloe barbadensis Miller) is a perennial plant of liliacea family with turgid green leaves joined at the stem in a rosette pattern. Aloe vera leaves are formed by a thick epidermis (skin) covered with cuticle surrounding the mesophyll, which can be differentiated into chlorenchyma cells and thinner walled cells forming the parenchyma (fillet). The parenchyma cells contain a transparent mucilaginous jelly which is referred to as Aloe vera gel.

Potential use of aloe products often involves some type of processing, e.g. heating, dehydration and grinding. Processing may cause irreversible modifications to the polysaccharides, affecting their original structure which may promote important changes in the proposed physiological and pharmaceutical properties of these constituents. Processing of Aloe vera gel derived from the leaf pulp of the plant, has become a big industry worldwide due to the application in the food industry. It has been utilized as a resource of functional food, especially for the preparation of health drinks which contain Aloe vera gel and which have no laxative effects. It is also used in other food products, for example, milk, ice cream confectionery and so on. However, Aloe vera gel juice was not very popular due to their laxative effect and majority of them contained absolutely no active mucilaginous polysaccharides or acemannan. Although colour changes have little relation to the therapeutic effectiveness of stabilized gel, they are rarely acceptable psychologically to the user. The color change is totally unacceptable in some products. It therefore becomes imperative that a simple but efficient processing technique needs to be developed, especially in the aloe beverage industry, to improve product quality, to preserve and maintain almost all of the bioactive chemical entities naturally present in the plant during processing.

The production process of aloe products involve crushing, grinding or pressing of the entire leaf of the Aloe vera plant to produce an Aloe vera juice, followed by various steps of filtration and stabilization of the juice. The resulting solution is then incorporated in or mixed with other solutions or agents to produce a pharmaceutical, cosmetic or food product.

In the food industry, Aloe vera has been utilized as a resource of functional food, especially for the preparation of health food drinks and other beverages, including tea. The amount of Aloe vera that finds its application in the pharmaceutical industry in not negligible as far as the manufacturing of topical ointments, gel preparations, tablets and capsules are concerned. Aloe vera gel also finds its application in the cosmetic and toiletry industries, where it is used as a base for the preparation of creams, lotions, soaps, shampoos and facial cleaners.

Unfortunately, because of improper processing procedures, many of these so-called aloe products contain, very little or virtually no active ingredients,

Corresponding Author: C.T. Ramachandra, Department of Agricultural and Food Engineering, Indian Institute of Technology, Kharagpur-721302, India Tel: +919449627325 Fax: +913222282244 
namely, mucopolysaccharides. In view of the known wide spectrum of biological activities possessed by the leaves of the Aloe vera plant and its wide spread use, it has become imperative that the leaf be processed with the aim of retaining essential bioactive components.

The review aims to provide a succinct resume of information regarding Aloe vara to serve as a reference for further investigations about this potential ingredient, to develop an effective method for processing of Aloe vera leaf, in the process, preserve and maintain almost all of the bioactive chemical entities naturally present in the Aloe vera leaf. The analysis deals with biological activity of leaf gel, gel stabilization technique, heat treatment of leaf gel, processing methodologies like cold-process, whole leaf process Qmatrix process, activealoe process, desiccant air dehydration, total process Aloe vera and Time Temperature and Sanitation (TSS) process.

\section{BIOLOGICAL ACTIVITY OF ALOE VERA}

The controversy over the identity of the active substance(s) in Aloe vera has not been settled. Also, various mechanisms have been proposed for the alleged healing properties of Aloe vera. Since no single definitive active ingredient has been found, it is commonly suggested that there may be some synergitic action between the polysaccharide base and other components $^{[1]}$.

According to Mackee ${ }^{[2]}$, vitamin D was the healing agent, but Row and Parks ${ }^{[3]}$ reported the absence of vitamin D. Morton $^{[4]}$ suggested a theory stating the seeming efficacy of aloe pulp may be attributed to its high water content, i.e., 96\%+, providing a means of making water available for injured tissue without sealing it off from the air. This recovery would explain the instant soothing effect of Aloe vera gel has on burns, but would not account for the long term effect of healing. The action of Aloe vera is simply due to its moisturizing and emollient effects, hence, its use in cosmetics.

Various researchers reported that the effective components for wound healing may be tannic acid ${ }^{[5]}$ and a type of polysaccharide ${ }^{[6]}$. Other researchers have also reported anti-inflammatory effects of complex polysaccharides, glycoproteines and sulfated polysaccharides. However, there are many examples in the literature indicating that polysaccharides can exhibit pharmacological and physiological activities without help from other components.

It is therefore, logical that the mucilaginous gel of Aloe vera plant, which is essentially a polysaccharide, holds secrete to Aloe vera's medicinal properties. Many researchers such as Collins and Collins ${ }^{[7]}$, Fine and Brown $^{[8]}$ and $\mathrm{Crew}^{[9]}$ have attributed pain-relieving properties to Aloe vera gel.

It is virtually impossible to prevent contamination by the leaf exudates during commercial extraction of Aloe vera gel. It is also believed that the intact leaves anthraquinones and their derivatives may diffuse into the gel from the bundle sheath cells; this possibly supports the conclusion of Row et al. ${ }^{[10]}$, who states that the healing agent is passed from the rind into gel on standing.

Davis $^{[11]}$, using the conductor-orchestra concept, explains the relationship that exists among over 200 biologically active compounds within Aloe vera. One of these molecules, a polysaccharide and acts as the conductor that leads a symphony composed of 200+ biologically active compounds. Davis concluded that, as the conductor, the polysaccharide modulates the biological activity between the surrounding orchestra molecules to work synergitically.

In view of these findings, it has seen presumptuous for any scientific research to consider or even to postulate that any one substance is responsible for the biological activity seen in Aloe vera gel.

Unfortunately, it is not easy to differentiate between a good quality product and one that has been adulterated. Although price can be a guide-the more expensive the Aloe vera, the better the product-this does not always apply. In the end, the key to judging Aloe vera is by results.

The things that happens to make aloe products less desirable or cause it to become virtually non beneficial are stem from the harvesting of the leaves, processing and distribution of leaves. The freshly removed leaves must go directly into production or must be appropriately refrigerated to prevent a loss of biological activity, principally through the degradative decomposition of the gel matrix. The value of aloe further diminishes if the processing procedure applies too much heat for too long a time. Extended heating renders the product free from bacterial contamination but effectively destroys aloe's mucopolysaccharide and consequently reduces its efficacy ${ }^{[12]}$. For therapeutic purposes, the most efficacious Aloe vera is that derived from whole-leaf aloe and cold-processed.

Aloe is not just aloe because the manufacturer says so. To assure that an aloe product at a price worth paying and to achieve the desired results, it is recommended to look for International Aloe Science Council (IASC) certification seal on literature and packaging. Another way to ascertain whether an Aloe vera product has a high healing capacity is to find out 
the number of mucopolysaccharides (MPS) present. This is sometimes included on the labeling. The highest therapeutic value is found in product containing between 10,000 and 20,000 MPS per liter.

\section{GEL STABILIZATION TECHNIQUE}

Aloe vera gel is the mucilaginous jelly obtained from parenchyma cells of the Aloe vera plant. When exposed to air, the gel rapidly oxidizes, decomposes and looses much of its biological activities. Different researchers have described different processing techniques of the gel with regards to its sterilization and stabilization, i.e., cold processing or heat treatment. However, the fundamental principle underlying these processing techniques remains almost the same. Regardless of the relative quality of the plant, the best results are obtained when leaves are processed immediately after harvesting. This is because degradative decomposition of the gel matrix begins due to natural enzymatic reactions, as well as the growth of bacteria, due to the presence of oxygen.

The entire process involves washing the freshly harvested Aloe vera leaves in a suitable bactericide, followed by processing of the leaves to mechanically separate the gel matrix from the outer cortex. The separation of the gel from the leaf could be facilitated by the addition of cellulose dissolving compounds, e.g., cellulose. Thus, the aloe liquid obtained is treated with activated carbon to decolourize the liquid and remove aloin and anthraquinones, which have laxative effects. This is especially so if the stabilized gel is to be used as a drink formulation for internal use. The resultant liquid is then subjected to various steps of filtration, sterilization and stabilization. The stabilized liquid, thus, obtained could be concentrated to reduce the amount of water or, alternatively, almost all of the water removed to yield a powder.

In cold processing technique, the entire processing steps are accomplished without the application of heat. Coats $^{[13]}$ reported the use of enzymes, like glucose oxidase and catalase, to inhibit the growth of aerobic organisms within Aloe vera gel and, thereby, sterilize it. Other sterilization steps reported in the cold processing includes exposing the gel to ultraviolet light, followed by a micron filtration.

In the heat treatment processing, sterilization is achieved by subjecting the aloe liquid obtained from the activated carbon treatment to pasteurization at high temperature. Aloecorp ${ }^{[12]}$ has reported the biological activity of Aloe vera gel essentially remains intact when the gel is heated at $65^{\circ} \mathrm{C}$ for periods less than $15 \mathrm{~min}$. Extended periods or higher temperatures have resulted in greatly reduced activity levels. They, however, suggested that the best method of pasteurization is HTST (High Temperature Shot Time), followed by flash cooling to $5^{\circ} \mathrm{C}$ or below.

In all these processing techniques, stabilization can be achieved by the addition of preservatives and other additives. The use of sodium benzoate, potassium sorbate, citric acid, vitamin $\mathrm{E}$ in synergism and the resultant efficacy, has been reported.

\section{HEAT TREATMENT OF GEL}

Xiu Lian Chang et al. ${ }^{[14]}$ conducted research on the gel juice from Aloe vera to investigate the effects of heat treatment on bioactive substances including polysaccharide and barbaloin. The effect of methanol solvent on compositional variations of barbaloin was also taken into consideration. Results show that the polysaccharide from Aloe vera exhibited a maximal stability at $70^{\circ} \mathrm{C}$ decreasing either at higher or lower temperatures. Heating promoted a remarkable decrease in barbaloin content depending on temperature and time, more affected than polysaccharide of the gel juice from Aloe vera. Barbaloin is unstable when dissolved in methanol resulting in the transformation into a series of unidentified compounds, in addition to aloe emodin with the period of storage at $4^{\circ} \mathrm{C}$ in refrigerator.

The effect of air-drying temperature (from 30 to $80^{\circ} \mathrm{C}$ ) on dehydration curves and functional properties (water retention capacity, WRC; swelling, SW; fat adsorption capacity, FAC) of Aloe vera cubes has been investigated by Simal et al. ${ }^{[15]}$ A diffusion model taking into account sample shrinkage has been proposed and solved by using a finite difference method. The effective diffusivities estimated with the proposed model varied with the air-drying temperature according to the Arrhenius law except for the experiment carried out at $80^{\circ} \mathrm{C}$, where case-hardening took place. Simulation of Aloe vera drying curves by using the model was accurate (percentage of explained variance (\%var): $99.7 \pm 0.1 \%$ ). Furthermore, drying kinetics of Aloe vera cubes of different sizes to those used to develop the model could be satisfactorily predicted (\%var: 99.5 $\pm 0.2 \%$ ). The three studied functional properties exhibited a maximum when drying temperature was $40^{\circ} \mathrm{C}$ decreasing either at higher or lower temperatures.

Physico-chemical modifications promoted by heat treatment and dehydration at different temperatures $\left(30-80^{\circ} \mathrm{C}\right)$ on acemannan, a bioactive polysaccharide from Aloe vera parenchyma were evaluated by Antoni Femenia et al. ${ }^{[16]}$ Modification of acemannan, a storage 
polysaccharide, was particularly significant when dehydration was performed above $60^{\circ} \mathrm{C}$. Heating promoted marked changes in the average molecular weight of the bioactive polysaccharide, increasing from $45 \mathrm{kDa}$, in fresh aloe, to $75 \mathrm{kDa}$, for samples dehydrated at 70 and $80^{\circ} \mathrm{C}$ respectively. The importance of physico-chemical modifications detected in dehydrated Aloe vera parenchyma depends on temperature used during the drying process. Regarding the chemical composition, the bioactive polysaccharide acemannan underwent similar losses of mannosyl residues when dehydration was performed between 30 and $60^{\circ} \mathrm{C}$, above the latter temperature, losses increased significantly. The physico-chemical alterations of the main type of polysaccharide may have important implications on the physiological activities attributed to the Aloe vera plant.

\section{PROCESSING OF ALOE VERA LEAVES}

Basic methods of processing Aloe vera leaves are

- Traditional hand filleted Aloe processing

- Whole leaf Aloe vera processing

- Total process Aloe vera processing

Traditional hand filleted aloe vera: In order to avoid contamination of internal fillet with the yellow sap, the traditional hand-filleting method of processing Aloe leaves was developed. In this method, the lower 1 inch of the leaf base (the white part attached to the large rosette stem of the plant), the tapering point (2-4 inch) of the leaf top and the short, sharp spines located along the leaf margins are removed by a sharp knife, then the knife, is introduced into the mucilage layer below the green rind avoiding the vascular bundles and the top rind is removed. The bottom rind is similarly removed and the rind parts, to which a significant amount of mucilage remains attached, are discarded. Another portion of the mucilage layer accumulated on the top of the filleting table. This is of critical concern because the highest concentration of potentially beneficial Aloe constituents are found in this mucilage, as this layer represents the constituents synthesized by the vascular bundle cells empowered by energy developed in the green (chlorophyll- containing) rind cells through suninduced photosynthesis.

The materials of the mucilage layer, subsequent to their synthesis, are distributed to the storage cells (cellulose-reinforced hexagons) of the fillet, a process that is accompanied by dilution owing to the water (the major fillet constituent), which is stored in the fillet cells. The fillet consists of more than $99 \%$ water. The fillet is washed again ensuring that there is no possibility of bacterial contamination, after which, the fillet is inserted into the pulper. The pulper has a refrigerated system that reduces the temperature of the resulting juice for optimum conversion, when the holding tank is full; it is left for $24 \mathrm{~h}$ to decant. Each tank is scientifically analyzed and certified, which takes approximately $170 \mathrm{~h}$. The way the inner gel is extracted from the leaf is very important. As mentioned above, the latex portion of the leaf is located between the rind and the inner gel. The gel should be removed from the leaf without disrupting this area so that little or no latex (aloin) gets into the gel. If latex does get into the gel, it makes the gel very bitter. This bitter taste can be distinguished from the vegetable taste of the inner gel with little experience. Just because Aloe juice is bitter, it does not mean that it contains 100\% pure Aloe juice from the inner fillet. If the gel is extracted by mechanical methods, the Latex can mix with the inner gel resulting in a loss in purity. Only by hand filleting the leaf it is able to cleanly separate the gel from the rind. The gel is then ground to a liquid and the pulp is removed. All this is performed at the farm, so only freshest leaves are processed.

The hand filleting method is very labour intensive. Owing to this fact, machines have been designed and employed which attempt to simulate the hand filleted techniques, but generally the product contains higher amounts of the anthraquinones laxatives than the traditional hand filleted approach.

Whole leaf aloe vera processing: This whole leaf process employed in the making of aloe juice allows the cellulose (skin) to be dissolved, as well as measurable amounts of aloin is to be removed. This total procedure is done entirely by a cold process treatment. Maximum efficiency is thus assured, resulting in a product rich in polysaccharides.

In this process, the base and tip are removed as previously delineated and then the leaf is cut into sections and ground into particulate slurry. The method for producing whole leaf Aloe vera begins by placing the whole leaf in a Fitz Mill grinding unit that pulverizes the entire leaf into a soup-like structure (Fig. 1). The material is then treated with, special chemical products which break down the hexagonal structure of the fillet releasing the constituents, by means of a series of coarse and screening filters, or passage through a juice press, the rind particles are removed, the expressed juice is then passed through various filtering columns which remove the undesirable laxative agents. This liquid is then pumped into large, stainless steel holding tanks that have been thoroughly cleaned and sanitized. 
Am. J. Agril. \& Biol. Sci., 3 (2): 502-510, 2008

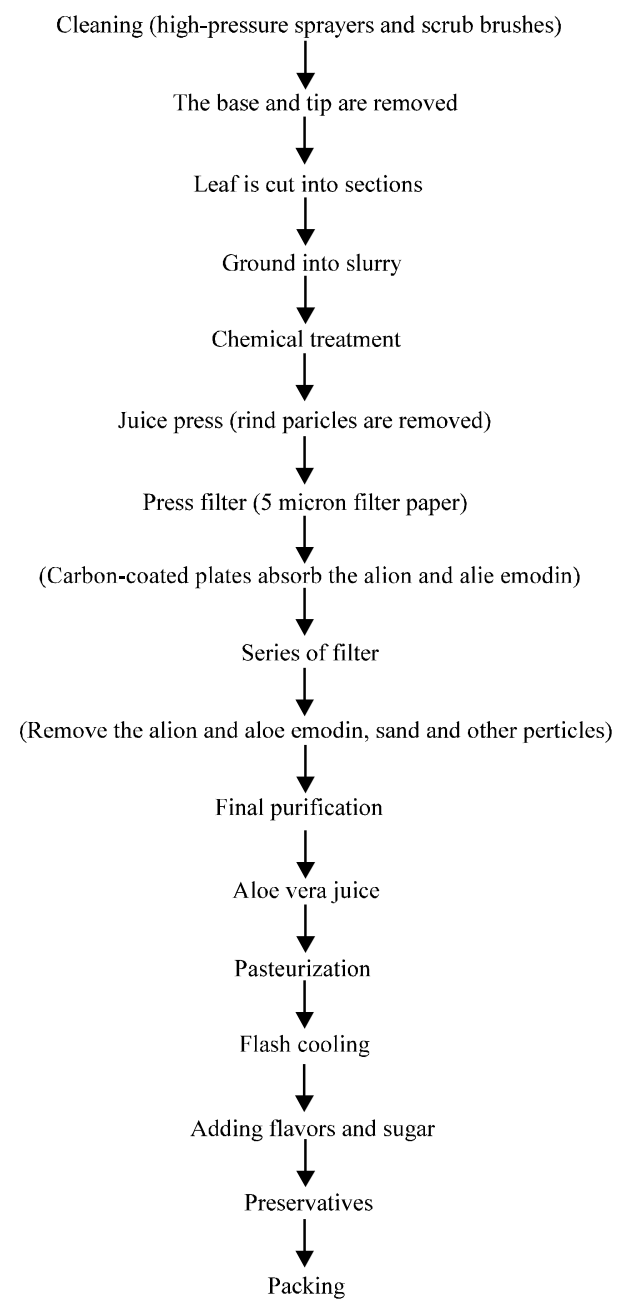

Fig. 1: Process flow diagram for whole leaf aloe vera processing

Once the tank is filled, it is hooked-up to a depulping extractor. This machine removes the large pieces of pulp and leaves that the initial grinding process developed. The result is the separation of the Aloe vera liquid and the pulp, which consists of the particles of Aloe leaf that have been ground and the naturally occurring pulp in the Aloe gel. The second phase of processing consists of passing the Aloe liquid through a series of filters that remove the aloin and aloe emodin (bitter-tasting, harsh laxatives) as well as any microscopic traces of leaves, sand or other particles.

A press filter is used during this phase. First, the press filter is attached to the storage tank containing the pre-filtered Aloe liquid. The press filter's carbon-coated plates absorb the aloin and aloe emodin that is a byproduct of grinding the whole leaf. The Aloe liquid is continually passed through the filter press until $99 \%$ of the aloin and aloe emodin are removed. This filtered product is then placed in a second holding tank.

At this point, a press filter containing five micron filter paper is attached to this holding tank. The Aloe liquid is passed through this filter medium until it shows no signs of residue. Cold filtration processing is then done as final purification procedure before the Aloe liquid is ready for stabilization.

This process, performed properly, can produce a constituent-rich juice (generally containing three times or more constituents than hand filleted juice), which should be virtually free from the laxative anthraquinones, this process was developed in the 1980's.

Total process aloe vera processing: In this new revolutionary approach, The Aloe leaves are hand filleted by the traditional, old fashioned, labour intensive method. Then the green rinds and the mucilage layer from the tabletop are processed by a newly developed propriety methodology. A combination of the products produced by these two procedures produces an aloe product called Total Process Aloe, which contains an enviably high concentration of desirable constituents, which are virtually free from undesirable laxative anthraquinones.

The traditional Hand-Fillet methodology, coupled with the newly developed proprietary handling of the refuse of the traditional methods (green rinds and tabletop mucilage) and a geographical area where aloe plants thrive have been combined in achieving the superior quality of Total Process Aloe. Total Process Aloe contains considerably higher concentrations of total solids, calcium, magnesium and malic acid, the major parameters of quality utilized and recommended by the International Aloe Science Council (IASC) for certification.

\section{MAJOR UNIT OPERATIONS IN PROCESSING OF ALOE VERA LEAF GEL}

Reception of raw materials: The Aloe vera leaves after harvesting were preferably transported in refrigerated vans from the field to the processing place. The leaves should be sound, undamaged, mold/rot free and matured (3-4 years) in order to keep all the active ingredients in full concentration ${ }^{[17]}$. However, the composition of these active ingredients are subtly affected by seasonal, climatic and soil variations. One important factor that must be considered is the handling/treatment of the leaves after its harvesting because the decomposition of the gel matrix occurs on 
cutting due to natural enzymatic reactions and the activity of bacteria that are normally present on the leaves. This degradative process can adversely affect the quality of the end product. Therefore, there is a need to carefully work towards refrigerating the freshly removed leaves within 4-6 $\mathrm{h}$ or get the raw material directly into production. Some information regarding the quality of a batch of Aloe leaves can be obtained by visual inspection (Fig. 2).

Filleting operation: The losses of biological activity appeared to be the result of enzymatic activity after the aloe leaf was removed from the plant. In fact, it was shown that the aloe gel, once extracted from the leaf, had greater stability than the gel left in the leaf. In order to avoid the decomposition of the biological activity, the filleting operation must be completed within $36 \mathrm{~h}$ of harvesting the leaves ${ }^{[18]}$. In the other hand, the anthraquinone was one important factor leading to nonenzymatic browning in aloe gel product ${ }^{[19]}$.

Grinding/homogenization: The major steps in this process include crushing or grinding. The aloe gel fillets should be crushed and homogenized using a commercial high speed tissue crusher at room

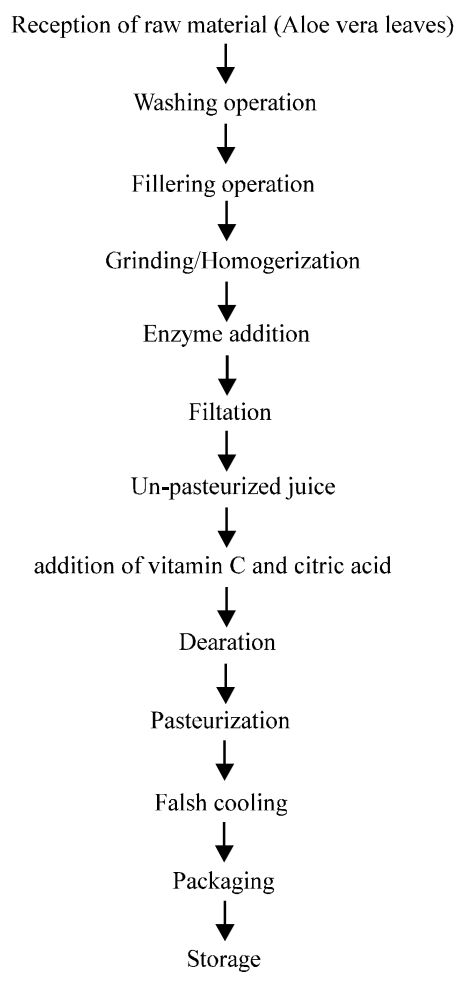

Fig. 2: Processing flow diagram of single-strength Aloe vera gel juice production temperature $\left(25^{\circ} \mathrm{C}\right)$. Due to the reaction of enzymatic browning, the longer the crushing/grinding time, the higher the browning index in Aloe vera gel juice ${ }^{[20]}$. Therefore, crushing or grinding should be shortened within 10-20 min in order to avoid the enzymatic browning reaction of Aloe vera gel.

Addition of pectolytic enzyme: Enzymatic treatment of Aloe vera gel for a long duration prior to processing is detrimental to biologically active compound such as polysaccharide which is the single most important constituent in aloe. Many researches have been done on the polysaccharides ${ }^{[21,22,23]}$. It has been reported that the enzyme treatment at $50^{\circ} \mathrm{C}$ and within $20 \mathrm{~min}$ did not induce the loss of biological activity of polysaccharide in Aloe vera gel ${ }^{[24]}$.

Filtration: This operation influences on the stability of Aloe vera gel juice. For example, the product showed the sedimentation of particles as the filtration operation lost its control.

Addition of vitamin $C$ and citric acid: The unpasteurized aloe gel juice was fortified with vitamin $\mathrm{C}$ and citric acid to avoid browning reaction, to improve the flavor of Aloe vera gel juice and to stabilize the juice ${ }^{[25,26,27,28]}$. The $\mathrm{pH}$ of aloe gel juice was adjusted between 3.0 and 3.5 by adding citric acid to improve the flavour of Aloe vera gel juice.

Dearation: The aim of dearation step is to avoid the oxidation of ascorbic acid ${ }^{[29]}$, which eventually improves the shelf life of the Aloe vera gel juice.

Pasteurization: Like the process of other vegetable juice, this step may affect the taste, appearance and the content of biological activity of aloe gel product. HTST treatment (at $85-95^{\circ} \mathrm{C}$ for $1-2 \mathrm{~min}$ ) is an effective method to avoid the bad flavour and the loss of biological activity of the Aloe vera gel ${ }^{[30]}$.

Flash cooling: After pasteurization, the juice is flash cooled to $5^{\circ} \mathrm{C}$ or below within $10-15 \mathrm{sec}$. This is a crucial step to preserve biological activity of the Aloe vera gel $^{[30]}$.

Storage: Relative humidity and temperature are two most important environmental parameters that affect product quality. Those two parameters can also affect the amount of the volatile substances of the juice absorbed by the packaging material ${ }^{[31]}$ and consequently, affect the shelf-life of the product ${ }^{[32,33]}$. 
Am. J. Agril. \& Biol. Sci., 3 (2): 502-510, 2008

\section{TIME TEMPERATURE AND SANITATION (TTS) PROCESS}

The stages of this innovative process technology is discussed hereunder

Timing of leaf process: Leaves show losses of biological activity beginning at $6 \mathrm{~h}$ following the harvest when the leaves are stored at ambient temperatures. A decrease in activity is also evident when the leaves are stored refrigerated, even though the rate of activity loss is greatly reduced. The losses of activity appear to be result of enzymatic activity after the leaf is removed from the plant. In fact it has been shown that the gel, once extracted from the leaf, has greater stability than gel which is left in the leaf. This means that shipping of leaves, even at refrigerated temperatures, will result in loss of biological activity. The overall timing of TTS production phase are extremely critical. The processing must be completed within $36 \mathrm{~h}$ of harvesting of leaves.

Leaf harvesting and handling: Biological activity is also due to the microbial decay of the gel. The first exposure of the inner gel to microbes is when the leaves are harvested from the plant. Leaves in which the base is not intact and sealed will greatly increase the microbial counts in the finished product. To prevent contamination of the gel, the leaves are handled carefully and soaked in a food grade sanitizer which effectively reduces the microbial count in the leaf exterior to acceptable levels.

Flash cooling: As a crucial step to preserve biological activity, the gel should be cooled below $5^{\circ} \mathrm{C}$ in 10 to $15 \mathrm{sec}$ following the gel extraction. Rapid cooling leads to enzymatic and microbial deterioration of the gel, but also aids in reducing the microbial counts in the product.

Pasteurization: Biological activity remains active when the gel is heated at $65^{\circ} \mathrm{C}$ for periods of less than 15 min. Extended periods or higher temperatures will results in greatly reduced activity levels. The best method of pasteurization is HTST (High Temperature Short Time), which expose the gel to elevated temperatures for periods of 1 to $3 \mathrm{~min}$. Once heated the gel is flash cooled to $5^{\circ} \mathrm{C}$ or below.

Concentration: The gel obtained using the pasteurization and flash cooling methods can be concentrated under vacuum without the loss of biological activity. The concentration operation must be conducted under $125 \mathrm{~mm}$ mercury vacuum at temperature below $50^{\circ} \mathrm{C}$ and must not exceed 2 min. Higher vacuum and temperature will cause activity loss, as will extend concentration times.

Freeze or spray drying: The concentrated product can then be freeze-dried at temperature between -45 and $30^{\circ} \mathrm{C}$ or can be spray dried with product temperature below $60^{\circ} \mathrm{C}$ without the loss in biological activity.

\section{DESICCANT DEHYDRATION PROCESS}

This system employs a low-tech procedure used for many years to dehydrate foods. The pure intact aloe fillets are first washed so that the first remaining aloin is removed. Then they are placed into a desiccant dehydration chamber where desired level of relative humidity and temperatures are maintained. Here the desiccant air is passed over the fillets to dry them. They come out of the chamber looking a little like a loofah sponge. This material is then ground to powder and packed.

By using this several important objectives are achieved. There is no concentration of the aloe gel. There by eliminating one step of the process. When the aloe is gently dried in the natural fillet form, the macromolecules do not break down like they do with mechanical pressing. The result is when the powder is re-hydrated, it comes back to its natural slippery form it had inside the leaf. It is generally believed that these delicate macromolecules are responsible for many Aloe veras' proteins. Because there is no need to pre-treat or pre-concentrate the aloe, there are no residual preservatives present in the final powder.

\section{QMATRIX PROCESS (ALOECORP)}

Qmatrix drying is 4th generation dehydration technology, which also includes microwave and radio frequency drying. Microwave and radio frequency drying are not appropriate for aloe as they can deacetylate aloe polysaccharides and denature proteins. For high quality foods, freeze-drying is traditionally used but it is relatively expensive (up to 10 times that of forced air dryers) and is limited to relatively small throughputs. Spray drying can be used for large throughput but the quality of the resultant product is inferior to that produced by freeze-drying due to volatile losses and heat damage. The Qmatrix process is a novel proprietary method of dehydration in enabling the dehydration of aloe while maintaining its integrity with respect to flavour, colour and nutrients. It is comparable to freeze drying in quality aspects but 
without the high operation costs (http://www. aloecorp.com).

\section{Advantages of this process:}

- Unique in the Aloe industry

- Exclusive to Aloecorp

- Gentle low temperature/short time drying

- Superior sensory attributes retained (Academic study)

- Superior retention of nutrients and bioactivity (Academic study)

- Atmospheric pressure (no vacuum)

- $\quad$ Energy efficient (Green)

- Environmentally friendly (Green)

- Superior solubility characteristics

- New proprietary products due to versatility

Solubility analysis of Qmatrix process: Spray dried aloe gel powder solubility was compared with Qmatrix processed powder. Equal quantities of powders were added at the same time to the same volume of room temperature water. Spray dried aloe clumps and floats whereas Qmatrix processed powder immediately disperse and settle to the bottom of the vessel. After $15 \mathrm{sec}$ of gentle stirring the Qmatrix processed material is completely in solution while spray dried powder is still clumped on the surface.

\section{ACTIVEALOE PROCESS}

Activealoe is Aloe vera manufactured by a patented process, developed using bioactivity guided research. The unique characteristics are as follows:

- Polysaccharide guarantee of not less than $10 \%$ by weight solids

- Controlled digestion of polysaccharides to enhance bioactivity

- Rapid processing to prevent breakdown of bioactive components

- Extensively tested and proven biologically active

Development of Activealoe process: Univera Pharmaceuticals investigated the role molecular weight played in the biological activity of aloe polysaccharides in order to develop a processing method that would retain and enhance the biological activity of native aloe resulting in the patented processing methods now used exclusively by Aloecorp.

\section{CONCLUSIONS}

A review on processing of Aloe vera leaf gel has revealed Aloe vera as a highly potential functional and valuable ingredient that exhibits relatively impressive biological functions of great interest in cosmetic, pharmaceutical and food industries. It also revealed the present processing technologies viz., gel stabilization technique, biological activity of aloe leaf gel and the effect of heat treatment on various constituents of gel. The process technologies like desiccant dehydration of aloe cubes, Qmatrix process, low temperature short time heat treatment process, activealoe process, Time Temperature and Sanitation Process, Total Process Aloe vera are the potential innovative process technologies.

\section{REFERENCES}

1. Leung, A.Y., 1978. Aloe vera in cosmetics. Excelsa, 8: 65-68.

2. Mackee, G.M., 1938. X-ray and Radium in the Treatment of Diseases of the Skin. Lea and Febiger (Eds.). Philadelphia, PA, pp: 319-320.

3. Row, T.D. and L.M. Parks, 1941. Phytochemical study of aloe vera leaf. J. Am. Pharm. Assoc., 30: 262-266.

4. Mortan, J.F., 1961. Folk uses and commercial exploitation of Aloe leaf pulp. Econ. Bot., 15: 311319.

5. Freytag, A., 1954. Suggested role of traumatic acid in Aloe wound healing. Pharmzie, 9: 705 (PUBMED) (INFORTIEVE).

6. Kameyama, S., 1979. Wound healing composition from Aloe arborescens extracts. Jap. Patent, 7856995.

7. Collins, C.E. and C. Collins, 1935. Roentgen dermatics treated with fresh aloe leaf. Am. J. Roentgenol., 33: 396-397.

8. Fine, A.F. and S. Brown, 1938. Cultivation and clinical application of aloe vera leaf. Radiol., 31: 735-736.

9. Crewe, J.E., 1939. Aloe in the treatment of burns and scalds. Minnesota Med., 22: 538-539.

10. Row, T.D., B.K. Lovell and L.M. Parks, 1941. Further observations on the use of Aloe vera leaf in the treatment of third-degree X-ray reactions. J. Am. Pharm. Assoc., 30: 266-269.

11. Davis, R.H., 1997. Aloe vera-A Scientific Approach. Vantage Press Inc., New-York, USA, pp: 290-306.

12. http://www.aloecorp.com. 
13. Coats, B.C., 1979. Hypoallergenic stabilized aloe vera gel. US patent 4: 178,372.

14. Xiu, L.C., W. Changhai, F. Yongmei and L. Zhaopu, 2006. Effect of heat treatment and dehydration on bioactive polysaccharide acemannan and cell wall polymers from Aloe barbadensis Miller. J. Food Eng., (75) 2: 245-251.

15. Simal, S., A. Femenia, P. Llull and C. Rossell, 2000. Dehydration of aloe vera: Simulation of drying curves and evaluation of functional properties. J. Food Eng., 43: 109-114.

16. Antoni, F., G. Pablo, S. Susana and R. Carmen, 2003. Effect of heat treatment and dehydration on bioactive polysaccharide acemannan and cell wallpolymers from Aloe barbadensis Miller. Carbohydrate Polymers, 51: 397-405.

17. Lawless, J. and J. Allan, 2000. Aloe Vera--Natural Wonder Cure. Harper Collins Publishers. London.

18. Robert, H.D., 1997. Aloe vera: A Scientific Approach. Vantage Press, Inc. New York.

19. He, Q., C. Liu and T. Zhang, 2002. Study on noenzymatic browning of aloe products and its inhibition methods. Food Sci. (Chenses), 23(10): 53-56.

20. Liu, C., 2001. Study on preservatives in the aloe gel juice system. J. Wuxi University Light Ind. (Chenses), 20(5): 480-484.

21. Gowda, D., B. Neelisiddaiah and Y. Anjaneyalo, 1980. Structural studies of polysaccharides from Aloe saponaria and Aloe vanbalenii. Carbohydrate Res., 83: 402-405.

22. Waller, G.R., S. Mangiafica and C.R. Ritchey, 1978. A chemical investigation of Aloe barbadensis Miller. Proceedings of the Oklahoma Academy of Science, 58: 69-76.

23. Yagi, A., S. Shibata, I. Nishioka, S. Iwadre and Y. Ishida, 1982. Cardiac stimulant action of constituents of Aloe saponaria. J. Pharm. Sci., 71: 739-741.

24. Maughan, R.G., 1984. Method to increase colour fastness of stabilized aloe vera. US Patent, 4: 465,629 .
25. Eison-Perchonok, M.H. and T.W. Downes, 1982. Kinetics of ascorbic acid oxidation as a function of dissolved oxygen concentration and temperature. J. Food Sci., 47: 765-767, 773.

26. Kacem, B., R.F. Mathews, P.G. Grandall and J.A. Cornell, 1987. Nonenzymatic browning in aseptic packaged orange juice and orange drinks. Effect of amino acids, dearation and anaerobic storage. J. Food Sci., 52: 1665-1667, 1672.

27. Kennedy, F.C., Z.S. Rivera, L.L. Lloyd, F.P. Warner and K. Jumel, 1992. L-ascorbic acid stability in aseptically processed orange juice in tetra brick cartons and the effect of oxygen. Food Chem., 45: 327-331.

28. Tramell, D.J., D.E. Dalsis and C.T. Malone, 1986. Effect of oxygen on taste, ascorbic acid loss and browning for HTST-Pasteurized, single-strength orange juice. J. Food Sci., 51: 1021-1023.

29. Chan, H.T., Jr. and C.G. Cavaletto, 1986. Effects of dearation and storage temperature on quality of aseptically packaged guava puree. J. Food Sci., 51: 165-168.

30. Eshun, K., 2003. Studies on aloe vera gel: Its application in beverage preparation and quality assessment. Thesis submitted to Food Science and Technology School of Southern Yangtze University in partial fulfillment of the requirements for the Degree of Master of Science.

31. Hernadez, R.J. and J.R. Giacin, 1998. Factors affecting permeation, sorption and migration processes in package-product systems. In: Food storage stability, Boca Raton, CRC Press, pp: 269329.

32. Hirose, K., B. Harte, J.R.Giacin, J. Miltz and C. Stine, 1988. Sorption of d-limonene by ealant films and effects on mechanical properties. In: Food and Packaging Interactions; ACS Symposium Series (vol. 365).

33. Sadler, G.D. and R.J. Braddock, 1990. Oxygen permeability of low density polyethylene as a function of limonene absorption. An approach to modelling flavour (Scalping). J. Food Sci., 55: 587-590. 